\title{
LA FLORA VASCULAR ENDÉMICA EN EL ESTADO DE QUERÉTARO. I. ANÁLISIS NUMÉRICOS PRELIMINARES Y DEFINICIÓN DE ÁREAS DE CONCENTRACIÓN DE LAS ESPECIES DE DISTRIBUCIÓN RESTRINGIDA
}

\author{
Jerzy Rzedowski ${ }^{1}$, Graciela Calderón de Rzedowski y Sergio Zamudio \\ Instituto de Ecología, A.C., Centro Regional del Bajío, \\ Apdo. postal 386, 61600 Pátzcuaro, Michoacán, México. \\ 1Autor para la correspondencia: jerzy.rzedowski@inecol.edu.mx
}

\section{RESUMEN}

Con bases firmes, la flora vascular del estado de Querétaro se estima en poco más de 4000 especies. De éstas 1625 (entre especies y entidades subespecíficas) se han inventariado en forma crítica en 174 fascículos publicados de la Flora del Bajío y de Regiones Adyacentes. De los 1625 taxa, 588 (36.18\%) son de distribución vasta. De los 1037 restantes, 709 (43.63\%) sólo se registran de México y 328 (20.18\%) restringen su distribución a Megaméxico 1, Megaméxico 2 y Megaméxico 3; 45 especies $(2.76 \%)$ son endémicas a los límites del estado de Querétaro; 26 (1.6\%) no se conocen sino de Querétaro e Hidalgo. La Sierra Madre Oriental, el centro de México, la Altiplanicie Mexicana y el Eje Volcánico Transversal contribuyen respectivamente con 94 (5.78\%), 92 (5.66\%), $37(2.27 \%)$ y $13(0.8 \%)$ especies de distribución limitada a las correspondientes regiones del territorio de la República. Los siguientes sectores de Querétaro se identifican como particularmente diversos en cuanto a especies de repartición restringida al estado: a) porción queretana de la Sierra Madre Oriental, b) la región más seca del estado ubicada en el centro del mismo, c) el profundo cañón del río Moctezuma, d) la parte alta del cerro Zamorano.

Palabras clave: endemismo, flora, México, Querétaro.

\section{ABSTRACT}

The vascular flora of the state of Queretaro is now estimated in somewhat more than 4000 species. 1625 of these (among species and subspecific entities) have been critically 
treated in 174 published fascicles of the Flora del Bajío y de Regiones Adyacentes. Out of these 1625 taxa, 588 (36.18\%) are of wide distribution. Out of the remaining 1037, 709 (43.63\%) are only known from Mexico and 328 (20.18\%) limit their distribution to Megamexico 1, Megamexico 2 and Megamexico 3; 45 (2.76\%) species are endemic to the limits of the state of Queretaro: $26(1.6 \%)$ are only known from the states of Queretaro and Hidalgo. The Sierra Madre Oriental, central Mexico, the Mexican High Plateau and the Volcanic Transversal Belt contribute respectively with 94 (5.78\%), 92 (5.66\%), $37(2.27 \%)$ and $13(0.8 \%)$ species of distribution restricted to their respective regions of Mexico. The following parts of Querétaro are identified as particularly diverse in species endemic to the state limits: a) the Queretaran part of Sierra Madre Oriental, b) the driest region of the state located in its central part, c) the deep canyon of Moctezuma river, d) the highest part of Zamorano peak.

Key words: endemism, flora, Mexico, Querétaro.

\section{INTRODUCCIÓN}

Hace unos 30 años todavía la flora de Querétaro era una de las menos conocidas de México, pues parte de su territorio carecía de buenas vías de comunicación y por consiguiente no resultaba atractivo para la gran mayoría de los colectores.

En 1991 Argüelles et al. publicaron una lista basada en los acervos existentes hasta esa fecha en los principales herbarios mexicanos, en la cual se reconocen 2334 especies de plantas vasculares. En 1992 Zamudio et al. estimaron que la riqueza florística del estado debe contener unas 1000 entidades adicionales. Arreguín et al. (1997) le adscribieron 2421 especies, mientras que Villaseñor (2003) calculó su diversidad de Magnoliophyta en 2887. En la actualidad la base de datos del herbario del Centro Regional del Bajío del Instituto de Ecología, A.C. (IEB) revela la existencia de ejemplares pertenecientes a 3935 especies de Tracheophyta, por lo que el inventario real, sin mucha duda, debe superar (aunque probablemente no en forma substancial) las 4000. Este número equivale a cerca de la quinta parte de la riqueza total calculada para el territorio de la República Mexicana.

Dada la relativa pequeñez del territorio del estado de Querétaro, tal cantidad lo ubica entre las porciones moderadamente ricas en cuanto a su flora concierne, y en virtud de esta circunstancia, la presente contribución pretende definir la importancia que en su universo vegetal juegan los elementos de distribución restringida.

En esta primera parte se da cuenta de los resultados del análisis numérico del endemismo y se señalan las porciones del estado en las que se concentran 
las especies de distribución restringida a los límites del mismo. En un segundo artículo se pretende enumerar e ilustrar todas las plantas conocidas de área prácticamente restringida a los límites de Querétaro, indicando su ubicación geográfica y ecológica conocida, así como una estimación de su grado de vulnerabilidad a la extinción.

\section{ÁREA DE ESTUDIO}

El territorio del estado de Querétaro tiene una extensión aproximada de 11,800 $\mathrm{km}^{2}$ y se encuentra ubicado en el centro-este de la República Mexicana. Su superficie constituye una especie de transecto, en el cual cabe distinguir tres regiones fisiográficas: la Sierra Madre Oriental, que ocupa más de un tercio nororiental, el Eje Volcánico Transversal que participa en forma de una franja meridional relativamente angosta, y la Altiplanicie Mexicana que constituye la amplia porción intermedia (Fig. 1).

La variada topografía incluye llanuras salpicadas con numerosos cerros y pequeñas sierras de diversos tamaños y elevaciones, profundos cañones y barrancas excavadas por los ríos, así como grandes cuerpos montañosos, cuyo relieve particularmente abrupto se manifiesta sobre todo en la Sierra Madre Oriental, esencialmente formada por rocas sedimentarias marinas, mientras que en el resto de Querétaro prevalecen las de directo o indirecto origen volcánico. Las altitudes oscilan entre 270 y $3360 \mathrm{~m}$.

Los climas secos (BS de la clasificación de Köppen) prevalecen en la mayor parte del estado, les siguen en importancia los templados semihúmedos $(\mathrm{Cw})$. Sólo una pequeña fracción los presenta húmedos de las categorías $(\mathrm{A}) \mathrm{C}(\mathrm{m}),(\mathrm{A}) \mathrm{C}(\mathrm{fm})$ y C(m), de acuerdo con la misma clasificación modificada por García (1973). Las temperaturas medias anuales varían entre 14 y $25^{\circ} \mathrm{C}$ y la precipitación recibida en promedio anual es de 350 a $2500 \mathrm{~mm}$.

En concomitancia con lo anterior, los matorrales xerófilos en sus diferentes variantes predominan en la cubierta vegetal de Querétaro. No faltan los pastizales, así como encinares, bosques de coníferas y bosques tropicales caducifolios. Una extensión bastante reducida en el noreste ocupa el bosque mesófilo de montaña y una aún más pequeña el bosque tropical subcaducifolio. Las comunidades acuáticas y subacuáticas también tienen una representación más bien limitada, pues se restringen prácticamente al bosque de galería, a varios charcos temporales, escasas asociaciones riparias y habitantes de presas y bordos. 
Para una definición más amplia y detallada de los aspectos ambientales, así como de las características de la flora y de la vegetación del estado, cabe consultar el trabajo de Zamudio et al. (1992).

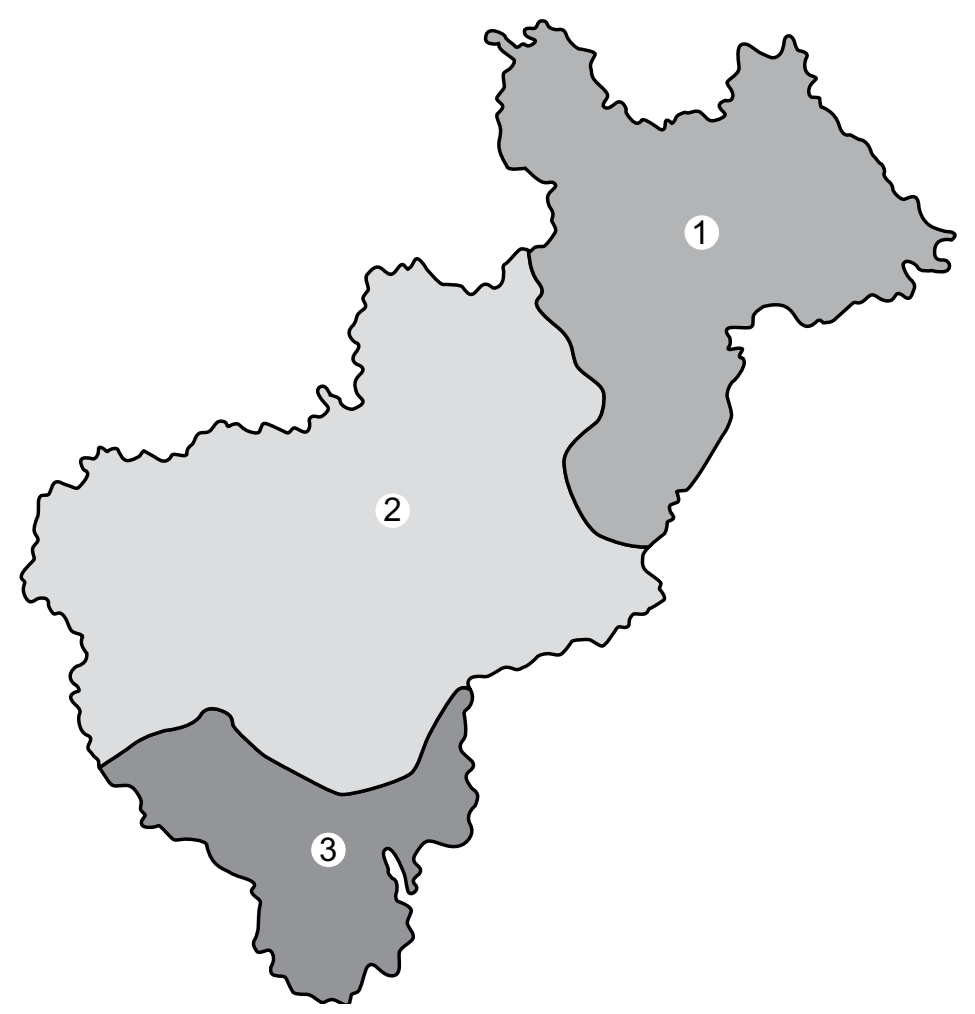

Fig. 1. Regiones fisiográficas del estado de Querétaro. 1. Sierra Madre Oriental. 2. Altiplanicie Mexicana. 3. Eje Volcánico Transversal.

\section{MÉTODOS}

Las principales herramientas del estudio empleadas en este trabajo son: los fascículos publicados de la Flora del Bajío y de Regiones Adyacentes, el acervo del herbario IEB (que registra 29,193 ejemplares colectados en Querétaro), la experiencia personal de los autores, que han recorrido prácticamente todos los rincones del estado, así como la consulta de la literatura y de la información disponible a través de internet. 
No se cuenta aún con el inventario cabal de la flora vascular de Querétaro, de manera que para este análisis se tomará esencialmente como base las especies habitantes del estado que ya han sido estudiadas en forma crítica y que aparecieron publicadas en los 174 fascículos de la Flora del Bajío y de Regiones Adyacentes. Tal conjunto que consiste de 1625 taxa, entre especies y entidades subespecíficas, constituye poco más de $40 \%$ del total de las 4000 estimadas para el estado, incluye miembros de todos los grupos importantes de plantas vasculares, por lo que puede considerarse como suficientemente representativo para que su análisis geográfico arroje proporciones similares a las de la totalidad de la flora.

\section{RESULTADOS}

Análisis geográfico de las especies de distribución restringida

Si se toma en consideración el área de repartición global conocida de los taxa, cabe calcular que 588 (36.18\%), son de distribución amplia o al menos más vasta que el área considerada como Megaméxico 3. También se sabe que 56 de ellas (3.44\%) son plantas introducidas de otras partes del mundo.

Las 1037 (63.81\%) restantes presentan mayor o menor grado de endemismo, para el cual se pueden reconocer las categorías establecidas en conexión con los principales objetivos de esta contribución (Cuadro 1). En la figura 2 se reproducen las áreas de referencia correspondientes a Megaméxico.

Los números del Cuadro 1 se prestan a los siguientes comentarios e interpretaciones:

a) La proporción de especies de amplia distribución (aprox. 36\%) es relativamente elevada entre las plantas vasculares de Querétaro, sobre todo si se le compara con la estimada por Rzedowski (1991a) para la flora fanerogámica del país entero, que es de aproximadamente $28 \%$. Tales números se revelan igualmente en los porcentajes de especies restringidas al territorio de México, que en Querétaro represen$\tan$ alrededor de $43 \%$, mientras que para todas las fanerógamas de la superficie de la República se calcularon en cerca de 52\%. Esta discrepancia refleja manifiestamente el hecho de que la porción oriental de nuestro país es substancialmente menos privilegiada en endemismos que la occidental, como ya lo señalaron varios autores, entre ellos Rzedowski (1991b). Otro factor que sin duda también interviene aquí es la circunstancia de que en los guarismos contados para Querétaro se incluyeron las pteridofitas, cuyas especies son mayormente de amplia distribución, así como las plantas introducidas. 
b) Dentro del conjunto de taxa endémicos a México prevalecen claramente los de distribución más general en el país, pero los restantes definen una notable vinculación con el oriente, observándose una importante proporción (5.78\%) de endemitas de la Sierra Madre Oriental, misma que contrasta con la escasa participación $(0.8 \%)$ de los propios del Eje Volcánico Transversal. Tal situación indudablemente está vinculada con la edad mucho más reciente de este último sistema montañoso.

c) A nivel de endemismo más estrecho resulta interesante observar que Querétaro descuella con más de $2.5 \%$ de especies de distribución prácticamente restringida

Cuadro 1. Categorías de la distribución total conocida de 836 especies de repartición limitada presentes en la flora vascular de Querétaro.

\begin{tabular}{|c|c|c|}
\hline Distribución total limitada a & Núm. de especies & $\%$ \\
\hline Querétaro* & 45 & 2.76 \\
\hline Querétaro e Hidalgo & 26 & 1.6 \\
\hline Querétaro y San Luis Potosí & 9 & 0.55 \\
\hline Querétaro y Guanajuato & 9 & 0.55 \\
\hline Eje Volcánico Transversal & 13 & 0.8 \\
\hline Altiplanicie Mexicana & 37 & 2.27 \\
\hline Centro de México & 92 & 5.66 \\
\hline Sierra Madre Oriental & 94 & 7.78 \\
\hline Este de México & 106 & 6.52 \\
\hline $\begin{array}{l}\text { México (sin corresponder a ninguno de los patrones } \\
\text { anteriores) }\end{array}$ & 199 & 12.24 \\
\hline México en total & 709 & 43.63 \\
\hline Megaméxico $1 * *$ & 116 & 7.13 \\
\hline Megaméxico 2 & 183 & 11.26 \\
\hline Megaméxico 3 & 29 & 1.78 \\
\hline Megaméxico en total & 328 & 20.18 \\
\hline Gran total & 1037 & 63.81 \\
\hline
\end{tabular}

*Puesto que varias de las especies aquí incluidas se conocen de lugares ubicados muy cerca de los límites del estado, es probable o ya conocido que también trascienden levemente las mencionadas fronteras políticas.

**Las categorías de Megaméxico 1, 2 y 3 se definen de acuerdo con el trabajo de Rzedowski (1991a). 
Rzedowski et al.: Flora vascular endémica en el estado de Querétaro
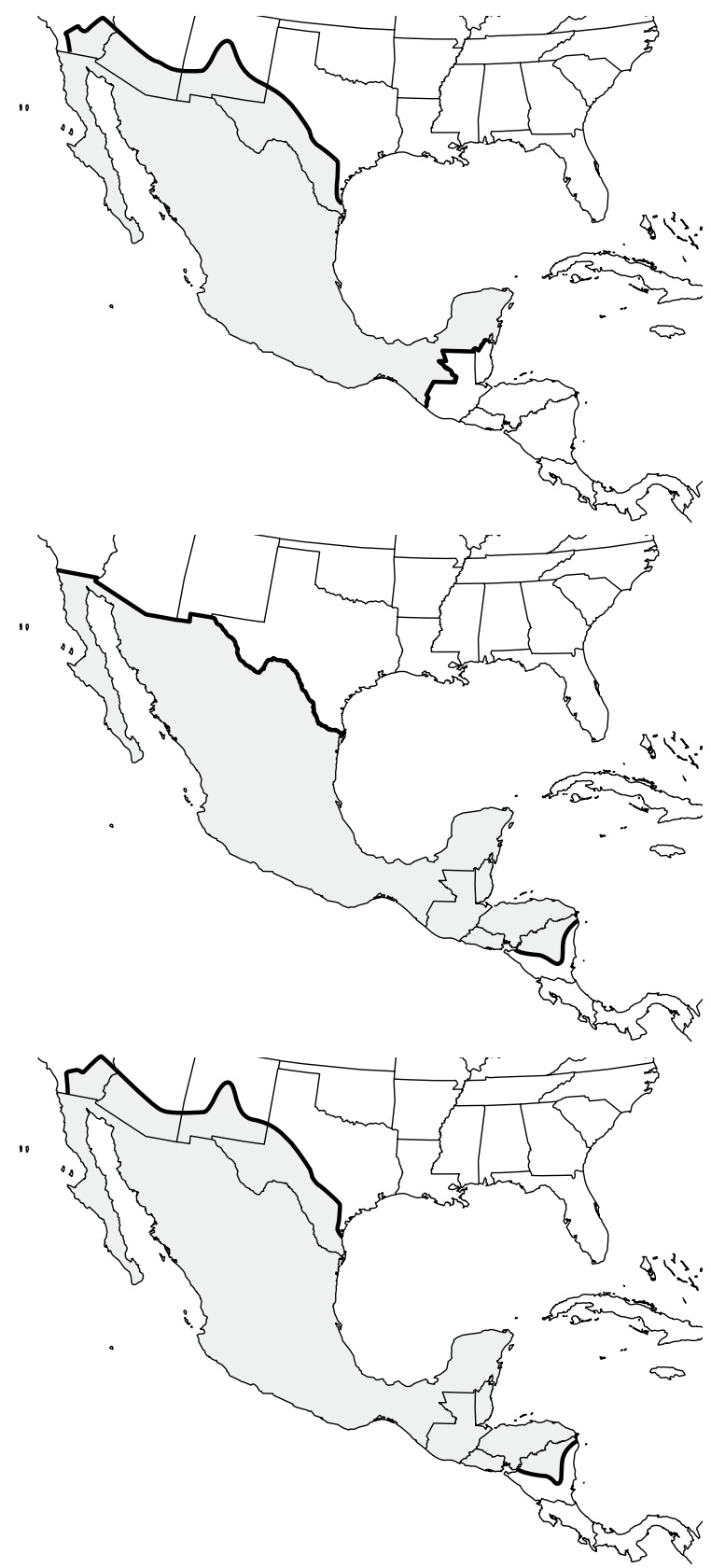

Fig. 2. Megaméxico. De arriba hacia abajo: Megaméxico1, Megaméxico 2 y Megaméxico 3, de acuerdo con el trabajo de Rzedowski (1991a). 
al estado (su enumeración puede consultarse en el Apéndice), número que se eleva a más de $5 \%$ si se suman las compartidas con cada una de tres entidades federativas vecinas (y subiría a ca. 7\% si se sumara el conjunto correspondiente a las que se comparten con dos o tres de ellos). Estos valores no comparan muy favorablemente con los dados a conocer para el Valle de Tehuacán-Cuicatlán (ca. 30\%), para la península de Baja California (23\%) y la de Yucatán (ca. 8\%) (Rzedowski, 1991b), o con $8.3 \%$ para el estado de Oaxaca (García-Mendoza, 2004), pero en proporción resultan significativamente superiores a la de $0.7 \%$ calculada para Morelos (BonillaBarbosa y Villaseñor, 2003), la de $0.9 \%$ registrada para Guanajuato (Zamudio y Galván, 2011) y las de muchos otros sectores de la parte central del país.

Áreas de concentración del endemismo vegetal

Zamudio et al. (1992) ya esbozaron la ubicación de las comarcas del estado particularmente diversas en cuanto a especies de plantas vasculares de repartición restringida. Éstas se ilustran en la figura 3 y a continuación se definen con mayor detalle.

1. El sector queretano de la Sierra Madre Oriental, que incluye los municipios de Arroyo Seco, Jalpan, Landa, así como partes de los de Pinal de Amoles, San Joaquín y Cadereyta, constituye la porción más rica en endemismos locales (25 spp., Apéndice). Es una superficie de aprox. $3500 \mathrm{~km}^{2}$, dominada por afloramientos de calizas. Una gran porción de esta comarca forma parte de la Reserva de la Biosfera de la Sierra Gorda. Presenta una cubierta vegetal variada, entre la cual destacan los bosques de encino y de coníferas, los tropicales caducifolios y subcaducifolios, los mesófilos de montaña, así como los matorrales submontanos.

2. La porción más seca del estado, situada en la parte central del mismo, integrada por importantes extensiones de los municipios de Tolimán, Peñamiller y Cadereyta, con una superficie aproximada de $3500 \mathrm{~km}^{2}$, con predominancia de calizas y lutitas como substrato geológico, es otra zona de particular concentración de especies endémicas (11 spp., Apéndice). Constituye una especie de isla ecológica de aridez más acentuada, en la cual prevalecen los matorrales xerófilos, en particular el micrófilo, el submontano y el rosetófilo.

3. El profundo cañón labrado por el río Moctezuma, desde el área actualmente ocupada por la presa Zimapán, hasta cerca del extremo oriental del municipio de Landa es el asiento de no pocas especies endémicas ( 5 spp., Apéndice). Su extensión territorial es difícil de definir y en toda su longitud señala el límite entre los estados de Querétaro y de Hidalgo, por lo que es más que probable la existencia de tales plantas en el territorio de esta última entidad federativa. Las especies de allí son 
habitantes de taludes horizontales o de laderas muy inclinadas de rocas calizas o lutitas. El sector correspondiente al municipio de Landa forma parte de la Reserva de la Biosfera de la Sierra Gorda; las porciones pertenecientes a los municipios de San Joaquín y de Cadereyta, que son las más importantes en cuanto a la ubicación de los endemismos, carecen de esta protección. En tal contexto cabe comentar que existen en la actualidad proyectos de convertir en presas adicionales a varios segmentos de tal región encañonada. También cabe agregar que esta parte del estado de Querétaro (y también de Hidalgo) no ha sido todavía suficientemente bien explorada y se espera localizar en ella otros endemitas adicionales.

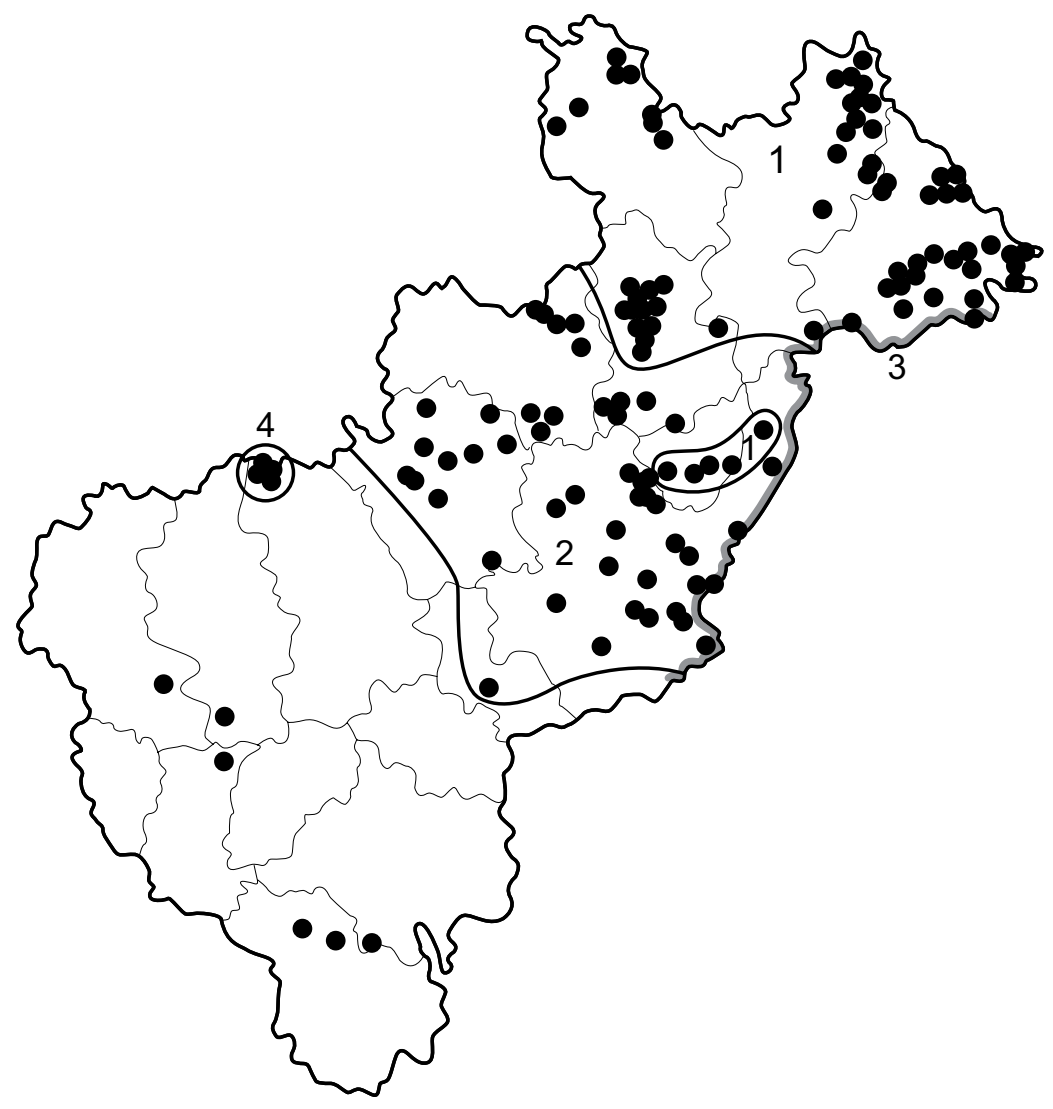

Fig. 3. Áreas de concentración de endemismo vegetal. 1. Sector queretano de la Sierra Madre Oriental. 2. La porción más seca del estado. 3. Cañón del río Moctezuma. 4. Parte alta del cerro Zamorano. 
4. Un centro adicional corresponde a la parte alta del cerro Zamorano, ubicado en el municipio de Colón y también en el de Tierra Blanca del estado de Guanajuato, pues su cresta que alcanza $3360 \mathrm{~m}$ de altitud, constituye el límite entre las dos entidades. Es un área de alrededor de $10 \mathrm{~km}^{2}$, cubierta mayormente por bosques de coníferas y de encino, que habitan sobre laderas fuerte a moderadamente inclinadas de rocas de origen volcánico. También se trata de una isla ecológica, pues por todos lados el macizo montañoso en cuestión se encuentra rodeado por grandes superficies de clima más seco. La porción guanajuatense fue declarada como Reserva de Conservación "Pinal de Zamorano", de aquí se registran tres especies endémicas.

A manera de conclusión y tomando en cuenta las cifras mostradas, así como el conocimiento de otros 32 endemismos ya registrados de familias aún no tratadas en la Flora del Bajío y de Regiones Adyacentes, cabe esperar que la cantidad total de especies de plantas vasculares restringidas o prácticamente confinadas al estado de Querétaro se incremente a cerca de un centenar.

Como ya se indicó, en la segunda parte de esta contribución se procurará proporcionar información sobre la ubicación geográfica de cada una de las especies endémicas ya conocidas.

\section{AGRADECIMIENTOS}

Los autores dan las gracias a Violeta Espinosa y a Alfonso Barbosa por la ayuda prestada en la confección y reproducción de las figuras. El trabajo fue realizado con apoyo del Instituto de Ecología, A.C. (cuenta 20006), del Consejo Nacional de Ciencia y Tecnología y de la Comisión para el Conocimiento y Uso de la Biodiversidad.

\section{LITERATURA CITADA}

Argüelles, E., R. Fernández y S. Zamudio. 1991. Listado florístico preliminar del estado de Querétaro. Flora del Bajío y de Regiones Adyacentes II: 1-155.

Arreguín S., M. L., L. Cabrera C., R. Fernández N., C. Orozco L., B. Rodríguez C. y M. Yepes B. 1997. Introducción a la flora del estado de Querétaro. Consejo de Ciencia y Tecnología del Estado de Querétaro. Querétaro, Qro. México. 361 pp.

Bonilla-Barbosa, J. R. y J. L. Villaseñor Ríos. 2003. Catálogo de la flora del estado de Morelos. Centro de Investigaciones Biológicas, Universidad Autónoma del Estado de Morelos. Cuernavaca, Mor., México. 129 pp. 
García, E. 1973. Modificaciones al sistema de clasificación climática de Köppen, para adaptarlo a las condiciones de la República Mexicana. 2a. ed. Universidad Nacional Autónoma de México. México, D.F., México. 246 pp.

García-Mendoza, A. 2004. Integración del conocimiento florístico del estado. In: GarcíaMendoza, A. J., M. J. Ordóñez y M. Briones-Salas (eds.). Biodiversidad de Oaxaca. Instituto de Biología, Universidad Nacional Autónoma de México - Fondo Oaxaqueño para la Conservación de la Naturaleza - World Wildlife Fundation. México, D.F., México. pp. 305-325.

Rzedowski, J. 1991a. Diversidad y orígenes de la flora fanerogámica del México. Acta Bot. Mex. 14: 3-21.

Rzedowski, J. 1991b. El endemismo de la flora fanerogámica mexicana: una apreciación analítica preliminar. Acta Bot. Mex. 15: 47-64.

Villaseñor, J. L. 2003. Diversidad y distribución de las Magnoliophyta de México. Interciencia 28: $160-167$.

Zamudio, S., J. Rzedowski, E. Carranza y G. Calderón de Rzedowski. 1992. La vegetación del estado de Querétaro. Consejo de Ciencia y Tecnología del Estado de Querétaro e Instituto de Ecología, A.C. Querétaro, Qro., México. 92 pp.

Zamudio, S. y R. Galván. 2011. La diversidad vegetal del estado de Guanajuato, México. Flora del Bajío y de Regiones Adyacentes XXVII: 1-101. 


\section{APÉNDICE}

Especies endémicas al estado de Querétaro registradas en 174 fascículos publicados de Flora del Bajío y regiones adyacentes.

Taxa

Justicia zamudioi T. F. Daniel

Ubicación

Anacardiaceae

Cotinus carranzae Rzed. \& Calderón

Sierra Madre Oriental

Aquifoliaceae

Ilex servinii E. Carranza

Sierra Madre Oriental

\section{Berberidaceae}

Berberis albicans Zamudio \& Marroq.

Zona árida

\section{Bromeliaceae}

Hechtia lepidophylla I. Ramírez

Zona árida

Hechtia zamudioi Espejo, López-Ferrari \& I. Ramírez

Zona árida

\section{Buxaceae}

Buxus moctezumae Köler, Fernández \& Zamudio

Río Moctezuma

\section{Campanulaceae}

Lobelia orientalis Rzed. \& Calderón

Sierra Madre Oriental

\section{Caprifoliaceae}

Abelia grandiflora Villarreal

Sierra Madre Oriental

\section{Commelinaceae}

Commelina queretaroensis López-Farrari, Espejo y Ceja

Sierra Madre Oriental

\section{Compositae}

Cirsium bicentenariale Rzed.

Sierra Madre Oriental*

Cirsium zamoranense Rzed.

Cerro Zamorano*

Coreopsis queretarensis B. L. Turner

Sierra Madre Oriental

Galinsoga elata Canne

Sierra Madre Oriental

Galinsoga glandulosa Canne

Sierra Madre Oriental

Perymenium arriagae Rzed. \& Calderón

Zona árida

Perymenium moctezumae Rzed. \& Calderón

Río Moctezuma

Perymenium sotoarenasii Rzed. \& Calderón

Zona árida 
Apéndice. Continuación.

\begin{tabular}{|c|c|}
\hline Taxa & Ubicación \\
\hline Tagetes moorei var. breviligulata Villarreal & Zona árida \\
\hline Tetrachyron omissum Rzed. \& Calderón & Sierra Madre Oriental \\
\hline Verbesina abietifolia Rzed. \& Calderón & Sierra Madre Oriental \\
\hline Verbesina carranzae P. Carrillo & Sierra Madre Oriental* \\
\hline Verbesina steinmannii P. Carrillo & Sierra Madre Oriental \\
\hline Viguiera paneroana Rzed. \& Calderón & Sierra Madre Oriental \\
\hline Zinnia zamudiana Calderón \& Rzed. & Zona árida \\
\hline \multicolumn{2}{|l|}{ Crassulaceae } \\
\hline Pachyphytum garciae E. Pérez-Calix \& Glass & Zona árida \\
\hline Sedum carinatifolium (R. T. Clausen) E. Pérez-Calix & Sierra Madre Oriental \\
\hline Sedum jerzedowskii E. Pérez-Calix & Sierra Madre Oriental \\
\hline \multicolumn{2}{|l|}{ Crossosomataceae } \\
\hline Velascoa recondita Calderón \& Rzed. & Sierra Madre Oriental \\
\hline \multicolumn{2}{|l|}{ Cucurbitaceae } \\
\hline Cyclanthera jeffreyi Lira \& Rodríguez-Arévalo & Sierra Madre Oriental \\
\hline \multicolumn{2}{|l|}{ Gentianaceae } \\
\hline Geniostemon rotundifolius Rzedowski \& Calderón & Sierra Madre Oriental \\
\hline \multicolumn{2}{|l|}{ Iridaceae } \\
\hline Sisyrinchium arguellesiae Ceja, Espejo \& López-Ferrari & Sierra Madre Oriental \\
\hline Tigridia rzedowskiana Aarón Rodr. \& L. Ortiz-Catedral & Sierra Madre Oriental \\
\hline \multicolumn{2}{|l|}{ Leguminosae } \\
\hline Acacia fusicarpa L. Rico & Zona árida \\
\hline \multicolumn{2}{|l|}{ Lentibulariaceae } \\
\hline Pinguicula calderoniae Zamudio & Sierra Madre Oriental \\
\hline Pinguicula elizabethiae Zamudio & Río Moctezuma* \\
\hline Pinguicula martinezii Zamudio & Sierra Madre Oriental \\
\hline Pinguicula moctezumae Zamudio \& R. Z. Ortega & Río Moctezuma* \\
\hline \multicolumn{2}{|l|}{ Malvaceae } \\
\hline Allowissadula glandulosa (Rose) D. M. Bates & Zona árida \\
\hline \multicolumn{2}{|l|}{ Rhamnaceae } \\
\hline $\begin{array}{l}\text { Colubrina macrocarpa var. macrocarpoides (Suess. ex Suess. } \\
\text { \& Overkott) M. C. Johnst. }\end{array}$ & Zona árida \\
\hline
\end{tabular}


Acta Botanica Mexicana 99: 91-104 (2012)

Apéndice. Continuación.

\begin{tabular}{|c|c|}
\hline Taxa & Ubicación \\
\hline \multicolumn{2}{|l|}{ Rosaceae } \\
\hline Potentilla queretarensis Rzed. \& Calderón & Sierra Madre Oriental \\
\hline Rubus macvaugianus Rzed. \& Calderón & Cerro Zamorano* \\
\hline \multicolumn{2}{|l|}{ Styracaceae } \\
\hline Styrax argenteus var. parvifolius E. Carranza & Sierra Madre Oriental \\
\hline \multicolumn{2}{|l|}{ Valerianaceae } \\
\hline Valeriana zamoranensis Rzed. \& Calderón & Cerro Zamorano* \\
\hline \multicolumn{2}{|l|}{ Violaceae } \\
\hline Viola cochranei H. E. Ballard & Eje Volcánico Transversal \\
\hline
\end{tabular}

